\title{
Bose-Einstein correlations in hadron-pairs from lepto-production on nuclei ranging from hydrogen to xenon
}

\author{
Gevorg Karyan** \\ Yerevan Physics Institute, A. Alikhanyan Br. 2, Yerevan, Armenia \\ DESY, Notkestrasse 85, Hamburg, Germany \\ E-mail: gevkardemail.desy.de
}

\begin{abstract}
Bose-Einstein correlations of like-sign charged hadrons produced in deep-inelastic electron and positron scattering are studied in the HERMES experiment using nuclear targets of ${ }^{1} \mathrm{H},{ }^{2} \mathrm{H},{ }^{3} \mathrm{H}$, ${ }^{4} H, N, N e, K r$, and $X e[1]$. A Gaussian approach is used to parametrize a two-particle correlation function determined from events with at least two charged hadrons of the same sign charge. This correlation function is compared to two different empirical distributions that do not include the Bose-Einstein correlations. Clear signals of Bose-Einstein correlations for all target nuclei without a significant variation with the nuclear target mass are found. Also, no evidence for a dependence on the invariant mass $W$ of the photon-nucleon system is found when the results are compared to those of previous experiments.
\end{abstract}

XXIV International Workshop on Deep-Inelastic Scattering and Related Subjects

11-15 April, 2016

DESY Hamburg, Germany

\footnotetext{
* Speaker.

${ }^{\dagger}$ For the HERMES Collaboration.
} 


\section{Introduction}

Semi-inclusive deep-inelastic scattering (SIDIS) of leptons from nuclei provides the tool to investigate a space-time evolution of a quark fragmentation into hadrons. In such a process, lepton transfers a certain amount of energy to the "struck" quark which then propagates through the nuclear medium. The distance scale over which a struck quark that received a sufficiently large energy-momentum transfer from an incident lepton develops into a colorless hadronic particle extends well beyond the size of a single nucleon leading to the modification of the final state hadron distributions due to the interaction with the nuclear medium. Thus a study of final state hadrons provides an access into the fragmentation process and its dynamics in nuclear environment. Furthermore, the size of a region in space-time from which bosons with similar momenta are emited can be studied in terms of intensity interferometry, a well-known technique developed by Hanbury Brown and Twiss to measure stellar radii[2]. In particular the Bose-Einstein correlations (BEC) of two identical bosons reflect both geometrical and dynamical properties of the particle radiating source. The BEC is a quantum mechanical phenomenon following from the symmetrization of a wave function of two integer spin particles leading to an enhanced probability for identical bosons to be emitted with small relative four-momentum compared with non identical bosons being in similar kinematic conditions. The schematic illustration of the Bose-Einstein effect is shown in Fig. 1., where a simple illustration of the emission and detection of two identical pions, from points $\mathbf{r}_{\alpha}$ and $\mathbf{r}_{\beta}$ is presented, which are observed with momenta $\mathbf{k}_{\mathbf{a}}$ and $\mathbf{k}_{\mathbf{b}}$ at detectors $a$ and $b$.

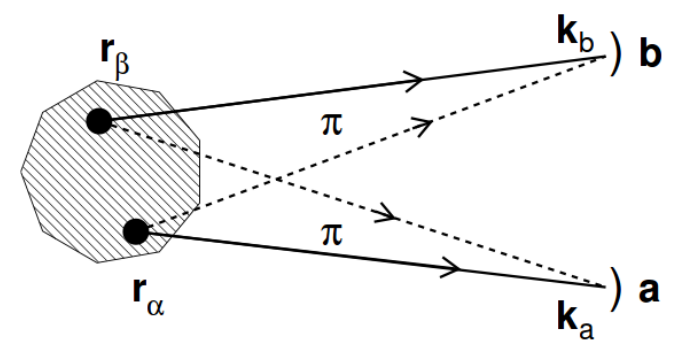

Figure 1: Schematic illustration of the Bose-Einstein effect.

The total wave function of two identical pion system can be symmetrized as follows :

$$
\Psi_{2 \pi}=\frac{1}{\sqrt{2}}\left(\Psi_{a \alpha} \Psi_{b \beta}+\Psi_{b \alpha} \Psi_{a \beta}\right)
$$

where $\Psi_{a \alpha}$ is the wave function of a pion produced at point $r_{\alpha}$ and observed at detector $a$ while $\Psi_{b \beta}$ is the wave function of a pion produced at point $r_{\beta}$ and observed at detector $b$. Under the assumption that the pion wave functions $\left(\Psi_{a \alpha}, \Psi_{b \beta}, \ldots\right)$ can be described by plane waves, i.e. $\Psi_{a \alpha} \approx e^{i \mathbf{k}_{\mathbf{a}} \mathbf{r}_{\alpha}}$, from the Eq. 1 one may obtain :

$$
\left|\Psi_{2 \pi}\right|^{2}=1+\cos (\delta \mathbf{k} \delta \mathbf{r})
$$

where $\delta \mathbf{k}=\mathbf{k}_{\mathbf{a}}-\mathbf{k}_{\mathbf{b}}$ and $\delta \mathbf{r}=\mathbf{r}_{\alpha}-\mathbf{r}_{\beta}$ are relative momentum and relative spacial distance respectively. Thus the correlation function resulting from the interference of two identical pion wave functions takes the following form : 


$$
R\left(\mathbf{k}_{\mathbf{a}}, \mathbf{k}_{\mathbf{b}}\right) \sim 1+\cos (\delta \mathbf{k} \delta \mathbf{r})
$$

\section{Experiment}

The data were collected with the HERMES spectrometer using $27.6 \mathrm{GeV}$ electron or positron beams stored in HERA at DESY[3].

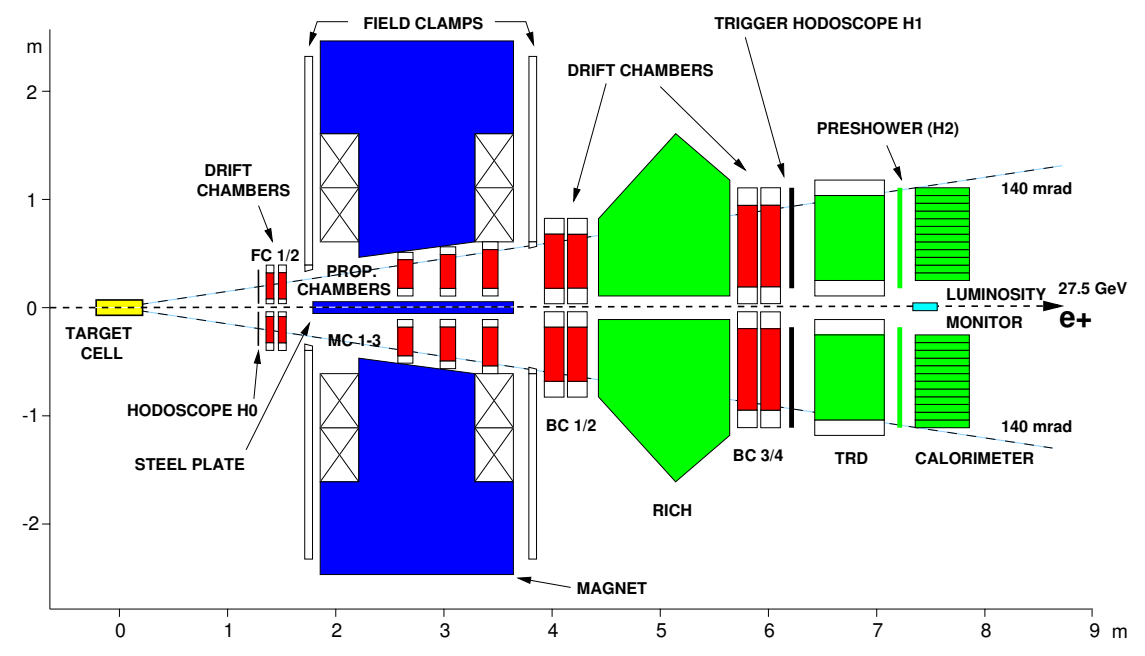

Figure 2: The HERMES spectrometer.

All the targets were internal to the lepton storage ring and consisted of polarized or unpolarized ${ }^{1} \mathrm{H}$, ${ }^{2} \mathrm{H}$ and ${ }^{3} \mathrm{H}$, or unpolarized ${ }^{4} \mathrm{H}, \mathrm{N}, \mathrm{Ne}, \mathrm{Kr}$, or $\mathrm{X} e$ gas injected into a thin-walled open-ended tubular storage cell. The trigger was formed by a coincidence between the signals from three scintillator hodoscope planes, and a lead-glass calorimeter where a minimum energy deposit of $3.5 \mathrm{GeV}(1.4$ $\mathrm{GeV}$ ) for unpolarized (polarized) target was required.

\section{Data analysis}

The analysed data sample is selected by imposing constraints on the squared four-momentum of the virtual photon $Q^{2}>1 \mathrm{GeV}^{2}$, and on the invariant mass of the photon-nucleon system $W^{2}>$ $10 \mathrm{GeV}^{2}$. For each event at least two charged hadrons with momenta $2.0 \mathrm{GeV}<p_{h}<15 \mathrm{GeV}$ required with only one identified lepton of the same charge as the beam lepton and momentum larger than $3.5 \mathrm{GeV}$. The measurement of the BEC effect in the experiment leads to the extraction of inclusive single particle spectrum in order to determine the probability density $D(p)$ which enters in the formal definition of the correlation function $R\left(p_{1}, p_{2}\right)$. A common practice is to substitute the two one-particle probability distributions $D\left(p_{1}\right) D\left(p_{2}\right)$ with a two-particle probability density reference distribution $D_{r}\left(p_{1}, p_{2}\right)$. The latter can be constructed from the experimental two-particle distributions that do not have any BECs. Taking into account this the experimental correlation can be written as :

$$
R\left(\mathbf{p}_{1}, \mathbf{p}_{2}\right)=\frac{D\left(p_{1}, p_{2}\right)}{D_{r}\left(p_{1}, p_{2}\right)}
$$


The simplest assumption is that the source has a symmetric Gaussian distribution which includes an additional normalization parameter $\gamma$ and a polynomial function $P(T)$ to describe the long-range correlations at large $T[4]$ :

$$
R(T)=\gamma\left[1+\lambda e^{-T^{2} r_{G}^{2}}\right] P(T)
$$

where $T=-\left(p_{1}-p_{2}\right)^{2}, r_{G}$ is the size of the particle source distribution and $\lambda$ is the chaoticity parameter. To construct the reference sample, two of the most widely used methods are taken :

a) Method of event mixing (MEM),

b) Method of unlike-sign pairs (MUS).

To construct the reference sample through $M E M$ hadron-pair distributions of the same charge are created by using hadrons from different events, while in $M U S$ hadrons with different charge from the same event are used. As an estimate of the systematic effects associated with these two reference samples PYTHIA-based Monte Carlo simulation of the HERMES experiment has been used. The quality of the HERMES simulation with respect to the description of the measured unlike-sign hadron pair distribution is demonstrated in Fig. 3 for a sample of hydrogen target data.

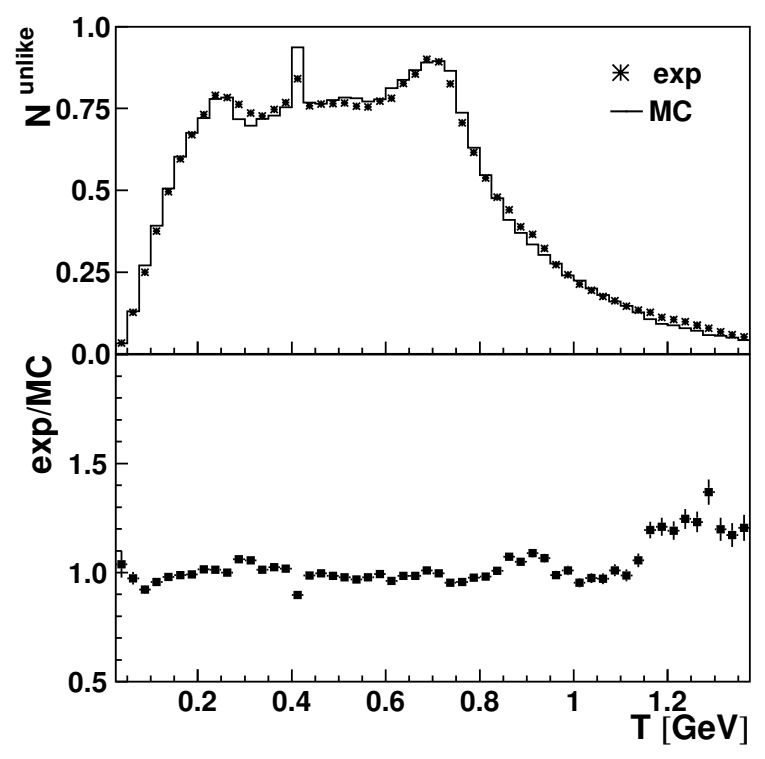

Figure 3: Top panel: normalized experimental (stars) and simulated (line) distributions for unlike-sign hadron pairs as a function of the variable $T$. Bottom panel: the ratio of these experimental (exp) and simulated (MC) distributions.

In order to reduce the biases resulting from the violation of kinematic constraints in the $M E M$ and from resonance contamination in the $M U S$ the experimental ratios are divided on the simulated ratios for two methods :

$$
\begin{aligned}
& R^{M E M}=(\text { like } / \text { mixed })^{\text {exp }} /(\text { like } / \text { mixed })^{M C} \\
& R^{M U S}=(\text { like } / \text { unlike })^{\text {exp }} /(\text { like } / \text { unlike })^{M C}
\end{aligned}
$$




\section{Results}

In Fig 4., the double-ratio correlation functions obtained from hydrogen data are shown by using two methods, i.e. $M E M$ and $M U S$, for extraction of the reference sample.

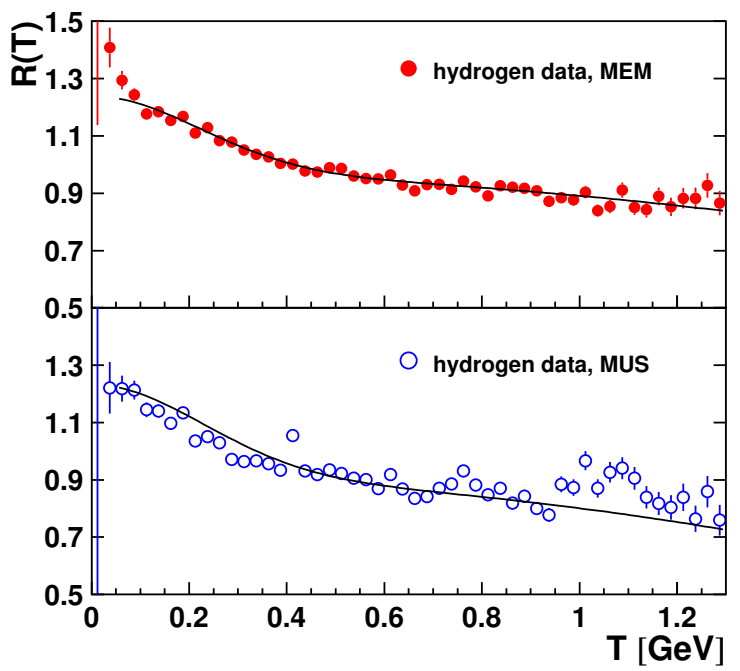

Figure 4: Double ratio correlation function for like-sign hadron pairs obtained with $M E M$ and $M U S$ based on hydrogen target data.

The Goldhaber parametrization (Eq. 5) is used to fit the data and the curves in the figure represent the fit. For MEM the extracted values of the fit parameters are : $r_{G}=0.64 \pm 0.03(\text { stat })_{-0.04}^{+0.04}(\mathrm{sys}) \mathrm{fm}$ and $\lambda=0.28 \pm 0.01(\text { stat })_{-0.05}^{+0.00}($ sys $)$ which is consistent with the results of the MUS: $r_{G}=$ $0.72 \pm 0.04(\text { stat })_{-0.09}^{+0.09}($ sys $) \mathrm{fm}, \lambda=0.28 \pm 0.02(\text { stat })_{-0.04}^{+0.02}($ sys $)$ within the present systematic and statistical uncertainties.
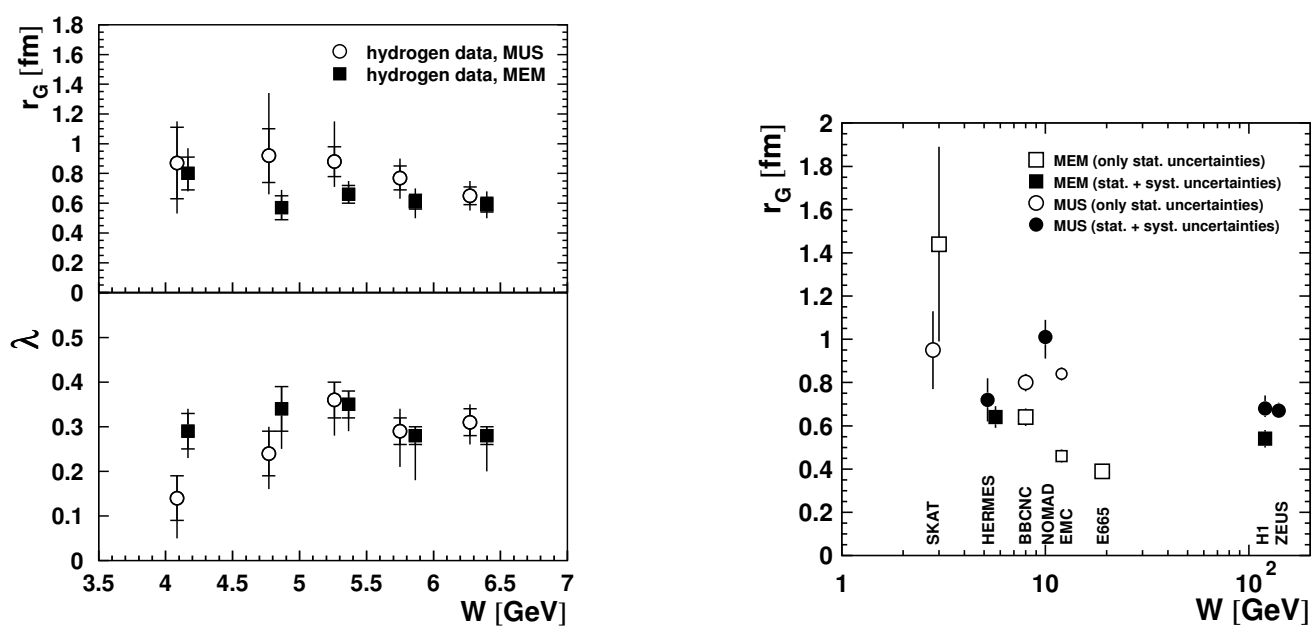

Figure 5: (left plot) Parameter $r_{G}$ (top panel) and $\lambda$ (bottom panel) as a function of $W$, obtained with $M E M$ and MUS methods on hydrogen. The inner and outer error bars indicate the statistical and total uncertainties. (right plot) Goldhaber radius $r_{G}$, as a function of $W$, obtained in lepton nucleon scattering experiments. 
In Fig. 5 (left plot), the parameters $r_{G}$ and $\lambda$ are presented for like-sign hadron pairs as a function of $W$ obtained with the $M E M$ and $M U S$ methods. There is no clear dependence of the parameters on the invariant mass $W$ observerd within the systematic and statistical uncertainties of the measurement. As one can see from Fig. 5 (right plot), the HERMES results on hydrogen are in general agreement with those of previous lepton-nucleon scattering experiments over a broad range in $W$, and agree well with the BBCN neutrino experiment [5], which is at a slightly higher mean $W$ than HERMES. There is also a good agreement observed with H1[6] and ZEUS[7] which are at much higher mean $W$.

To examine a possible nuclear dependence in BEC, the correlation function for like-sign hadron pairs produced in scattering off the nuclear targets ${ }^{2} \mathrm{H},{ }^{3} \mathrm{H},{ }^{4} \mathrm{H}, \mathrm{N}, \mathrm{Ne}, \mathrm{Kr}$, and $\mathrm{Xe}$ is measured using the same parametrization as given in Eq. 5 .

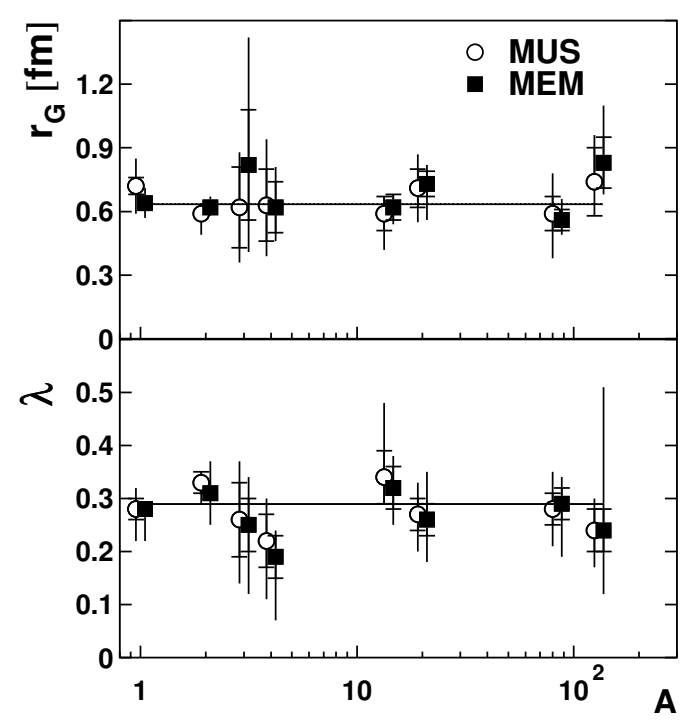

Figure 6: The parameters $r_{G}$ (top panel) and $\lambda$ (bottom panel) are shown as a function of the target atomic mass $A$. The inner part of the error bars indicate the statistical uncertainty and the total error bars have systematic uncertainties added in quadrature.

Within the estimated uncertainties no dependence of $r_{G}$ and $\lambda$ parameters on target atomic mass is observed.

\section{References}

[1] A. Airapetian et al, Eur. Phys. J. C 75 (2015) 361.

[2] R. Hanbury Brown and R.Q. Twiss, Nature 178, 1046 (1956).

[3] K. Akertaff et al., HERMES coll., Nucl. Instrum. Methods A417 239 (1998).

[4] G. Goldhaber, S. Goldhaber, W. Lee, A. Pais, Phys. Rev. 120, 300 (1960).

[5] BBCN Collaboration, V.A. Korotkov et al., Zeit. Phys. C 60, 37 (1993).

[6] H1 Collaboration, C. Adloff et al., Zeit. Phys. C 75, 437 (1997).

[7] ZEUS Collaboration, S. Chekanov et al., Phys. Lett. B 583, 231 (2004). 